Kinder in der "Muckibude"

\title{
„Beim Krafttraining ist die Gefahr der Überforderung minimal“
}

In Hamburg hat die deutschlandweit erste „Muckibude“ für Kinder eröffnet. Wir sprachen mit dem Sportwissenschaftler Priv.-Doz. Dr. Michael Fröhlich, Universität des Saarlandes, über Nutzen und Risiken von Krafttraining im Kindesalter und über den überraschenden Meinungsumschwung vieler Experten.

MMW: Herr Dr. Fröhlich, warum sollte man ein Kind Krafttraining machen lassen?

Fröhlich: Gezieltes Krafttraining kann die sportartspezifische Leistungsfähigkeit steigern. Das nützt z. B. Kindern, die in irgendeiner Sportart aktiv sind. Zum anderen wird durch adäquat angeleitete Übungen die allgemeine Muskulatur gekräftigt. Damit verbessert sich das Körpergefühl, die Haltung wird gerader, die Kinder bekommen ein positives Selbstbild. Eine gut ausgeprägte Muskulatur verbraucht auch mehr Energie. Deshalb ist das Krafttraining vor allem auch für übergewichtige Kinder mit geringerer Leistungsfähigkeit geeignet.

MMW: Was spricht dagegen, die Kinder einfach auf den Bolzplatz oder ins Ballett zu schicken?

Fröhlich: Das kann man natürlich auch machen. Adäquates Krafttraining ist eine sinnvolle Ergänzung. Aber es ist auch ein guter Einstieg, weil man Barrieren, die in verschiedenen Sportarten existieren, umschiffen kann. Kinder, die zu dick sind oder eine schlechte Haltung haben, werden in der Schule oft gehänselt. Die muss man nicht in einen Verein stecken, wo sie noch mehr als Außenseiter betrachtet werden. Beim Kraftsport gibt es weniger Ausgrenzungstendenzen. Die Kinder haben oft einen ähnlichen sozialen Hintergrund, vielleicht auch eine ähnliche Physiognomie. Man kann die Belastung individuell auf das Kind abstimmen und damit die
Gefahr der Überforderung minimieren. Vor allem stellt sich relativ schnell ein positiver Effekt in Form einer deutlichen Leistungssteigerung ein. Wenn die Kinder sehen, was sie erreicht haben, lassen sie sich vielleicht auch für andere sportliche Aktivitäten motivieren.

MMW: Lange war das Krafttraining für Kinder verpönt. Man warnte vor Verletzungen, Wirbelsäulen- und Knochendeformierungen. Heute sprechen sich viele Experten für das Krafttraining aus. Wie kommt es zu diesem Meinungsumschwung?

Fröhlich: Studien aus den 1980er-Jahren konnten keine nennenswerte Steigerung der Kraft nachweisen. Die Faktenlage war aber sehr dünn, die Studien beruhten auf kleinen Stichproben und arbeiteten mit geringer Belastungsintensität. Die American Academy of Pediatrics hat dann einfach den Sicherheitsaspekt in den Vordergrund gestellt und vor Kraftraining gewarnt. Spätere Längsschnittstudien konnten alle diese Befürchtungen, also schädlichen Einfluss auf den Entwicklungsprozess, Schädigung der Knochenstruktur, der Epiphysenfugen usw., ausräumen.

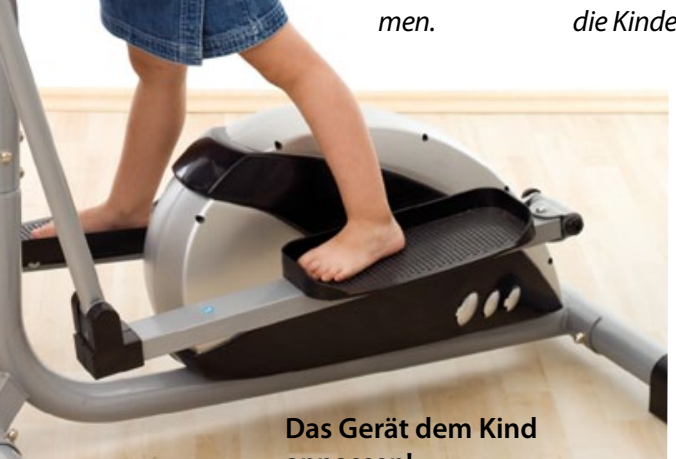
anpassen!

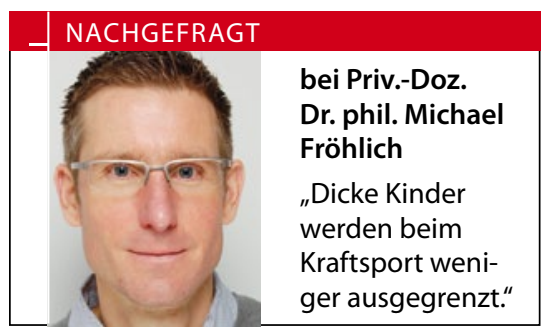

MMW: Kritisiert wurde ja auch, dass Kinder wegen ihrer niedrigeren Hormonspiegel gar nicht vom Krafttraining profitieren können.

Fröhlich: Tatsächlich sind bei Kindern Hormone wie Testosteron, Wachstumshormone, Insulin-like growth factor 1 etc. noch nicht in so hoher Konzentration vorhanden wie bei Erwachsenen. Nichtsdestotrotz führt das Training zu Anpassungen, z. B. zu einer Zunahme der Muskelmasse und zu einer Verbesserung der intra- und intermuskulären Koordination.

MMW: Ab welchem Alter kann man mit dem Krafttraining beginnen?

Fröhlich: Kindgerecht, spielerisch eingebettet als Partnerübungen, mit Therabändern oder Bällen kann das Training schon mit fünf, sechs Jahren einsetzen. Ein Gerätetraining kann man mit zehn, elf Jahren beginnen.

MMW: Worauf muss man achten, damit das Training dem Kind nicht schadet?

Fröhlich: Eher abraten würde ich von Übungen mit dem eigenen Körpergewicht, also Liegestützen, Klimmzügen oder Kniebeugen. Der Reiz ist dabei oft zu hoch, vor allem für übergewichtige Kinder. Ein Gerätetraining, mit dem man die Belastungsintensität individuell anpassen kann, ist viel günstiger. Die Geräte müssen kindgerecht sein, Hebelarme, Sitzpolster, Lendeneinstellung müssen sich adäquat positionieren lassen, es muss Gewichtsabstufungen geben, am besten im Halbkilobereich. Die Kinder müssen von professionellen, pädagogisch gut ausgebildeten Trainern unterwiesen werden. Die Belastung muss in Relation zum kindlichen Organismus festgelegt werden*. Ganz wichtig ist auch, dass die Kinder nicht den Spaß verlieren. Das Kind sollte in den Prozess des Trainings integriert werden. Nur so erreicht man, dass es auch dabei bleibt.

- Interview: Dr. Elke Oberhofer

* http://www.nsca-lift.org/WorkArea/ DownloadAsset.aspx?id=1155 